\title{
Effectiveness of 14 day quarantine strategy: Chinese experience of prevention and control
}

\author{
Tie Song and colleagues argue that a 14 day quarantine of inbound passengers is an effective \\ and proportionate measure to control imported covid-19
}

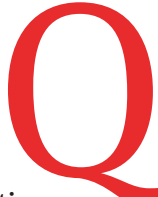

uarantine for inbound travellers has been considered an important measure to prevent imported cases of covid-19. The World Health Organization recommends that all contacts of people with confirmed or probable covid-19 should be quarantined in a designated facility or isolate at home for 14 days from their last exposure. ${ }^{1}$ However, the quarantine period for travellers has varied across different countries and regions. In countries such as the US and Greece travellers are required to isolate at home for just seven days, whereas in May 2021 Vietnam required arrivals to spend 21 days in a designated quarantine facility plus seven days of isolation at home (this was reduced to seven days at a designated facility and seven days at home in July 2021).

Countries with no or low local viral transmission such as China, Korea, and Singapore may benefit more from a 14 day quarantine rule than those with higher rates. $^{2}$ In China, a 14 day quarantine is combined with nucleic acid tests performed once on days 1, 4, and 7 and twice on day 14 , and the samples are tested by two different institutions using two types of

\section{KEY MESSAGES}

- 14 day quarantine of inbound travellers has been an important part of China's prevention and control strategy

- The incubation period of SARS-CoV-2 was estimated at 5-6 days, but is shorter for some variants

- Most people who develop symptoms will do so within 14 days, and asymptomatic people have weaker infectivity than those with symptoms

- Individuals who test positive after 14 days' quarantine account for less than $2 \%$ of imported PCR positive cases in China

- Vaccinated people can still become infected with SARS-CoV-2 and still require quarantine detection reagents. The quarantine can be lifted if all test results are negative. If the test result is positive or relevant symptoms develop, the individual will be transferred to a designated hospital.

As the pandemic continues to rage, local outbreaks caused by people with an extended incubation period or asymptomatic disease have been reported and more infectious strains (such as alpha and delta variants) have been found in multiple countries. As a result, some countries strengthened the controls of inbound passengers-for example, increasing quarantine from 14 days to 21 or even 28 days. Such extended quarantine measures are probably an over-reaction to the risk, but the looser controls in some countries or districts, where only social distance and simple protection measures were applied, may have contributed to the spread. We examine whether quarantine measures are effective and proportionate to control imported cases and prevent spread of the covid-19 epidemic.

\section{Incubation period of SARS-CoV-2}

SARS-CoV-2 seems to be most contagious around the time of symptom onset. ${ }^{3}$ Infectiousness starts two to three days before symptoms appear, peaks around a day before symptom onset, and falls rapidly within seven days after symptom onset. ${ }^{4}$ The incubation period of this virus is therefore a key factor in setting the duration of quarantine.

An early study in Hubei, China, estimated that the incubation period of SARS-CoV-2 was 5.1 days (95\% confidence interval 4.5 to 5.8 days), and $97.5 \%$ of infected people who developed symptoms did so within 11.5 days (8.2 to 15.6 days). However, the study also indicated that about $1 \%$ of infected people might take more than 14 days to develop symptoms. ${ }^{5}$ Another study found the best fitting distribution of the incubation period was a Weibull distribution, which showed a marked skewed distribution with a mean period of 6.4 days (median 5.7, interquartile range $3.2-8.8){ }^{6}$ A meta-analysis of peer reviewed studies of SARS-CoV-2 infection estimated the mean incubation period was 6.3 days (median 5.4, 95\% quantile 13.1). ${ }^{7}$ These studies have been used to inform the duration of quarantine.

Asymptomatic infections also account for around a third of all PCR (polymerase chain reaction) positive cases. ${ }^{8}$ A metaanalysis showed that the summary relative risk of secondary infection among contacts of asymptomatic people was 0.35 (95\% confidence interval 0.10 to 1.27 ) compared with symptomatic patients. ${ }^{9}$ This suggests that people with asymptomatic infections are unlikely to contribute greatly to the spread of covid-19. However, the clinical, immunological, and viral characteristics of asymptomatic people remain unclear, and further analyses are needed. China therefore continues to manage asymptomatic people in the same way as those with symptoms.

As understanding of SARS-CoV-2 grew, more attention was paid to the epidemic characteristics of different strains. Early epidemiological data show that SARSCoV-2 strains with the mutated D614G amino acid in the spike protein have more efficient replication and higher infectivity than the original virus, and these became the dominant form in several countries during the early stages of the epidemic (March-April 2020). ${ }^{10} 11$ Subsequently, variants with new mutations in the spike protein developed with stronger infectivity and adaptability, including alpha, beta, gamma, and delta. Evidence is increasing that these variants have shorter incubation periods and longer viral shedding. The median incubation period seems to be about three days for the alpha variant ${ }^{12}$ and four days for the delta variant, ${ }^{13} 14$ for example. However, information on these variants is still insufficient and more evidence is needed to provide data to set the quarantine time.

\section{China's experience of quarantine strategy}

Travel into China was restricted during the pandemic, and passengers entering Guangdong province accounted for around 90\% 
of all Chinese arrivals. From 1 May 2020 to 7 March 2021 there were 1868 imported cases of SARS-CoV-2 infection identified, but only $33(1.8 \%)$ people tested positive after 14 days' quarantine. Only three of these 33 people developed symptoms. Four were not tested for IgG antibody, but the positivity rate in the rest was $97 \%(28 / 29)$. This suggested these cases were not likely to be new infections but that the people had been infected while abroad and were testing positive again in China. Two of the three people with symptoms were IgG positive when they arrived in China and their cycle threshold values on nucleic acid testing were 30 and 38.4, respectively. Therefore, they were considered to be reinfections. As the positive results could be due to detection of degraded virus fragments and high levels of antibodies were observed, the infectivity of these cases may be extremely low. ${ }^{15}$

Epidemiological investigation revealed that the third confirmed case lived with someone who was PCR positive and developed covid-19 symptoms during the quarantine period. Therefore, this case was evaluated as a secondary infection of SARS-CoV-2 during the quarantine period. As those identified cooperated with other prevention and control measures, no local epidemic was induced by these three cases. This experience suggests the 14 day quarantine is sufficient.

Some researchers have also found longer quarantine times are not necessary to control the covid-19 pandemic. Ashcroft and colleagues found that 10 days' quarantine can prevent 99.9\% (95\% confidence interval $98 \%$ to $100 \%$ ) of local transmission caused by returned travellers. ${ }^{16}$ Another analysis suggested that a 14 day quarantine would result in fewer than one infectious traveller entering Britain from the EU or the US each week. ${ }^{17}$ These analyses suggest that although extending the 14 day quarantine may be worth considering in some extreme cases, a longer quarantine time may not add benefit when social-psychological and economic effects are taken into consideration. ${ }^{18}$

\section{Management of vaccinated travellers}

Several types of vaccines, including mRNA vaccines, inactivated vaccines, and adenovirus vaccines, have been listed for emergency use by WHO and are widely used around the world. However, no vaccine provides $100 \%$ protection against the SARS-CoV-2 virus, and vaccinated people still have the potential to carry the virus. ${ }^{19}$ ${ }^{20}$ Studies based on the SARS-CoV-2 vac- cine in macaques have shown that past infection or vaccination can protect from severe disease, but individuals may have similar potential to transmit the virus as those who have never been exposed to the virus. ${ }^{21}$ Moreover, recent modelling studies suggest that under the conditions of low vaccination coverage, detection and quarantine of inbound passengers needs to be maintained to avoid local outbreaks and overloading of healthcare facilities. ${ }^{22}$ As there is insufficient evidence to support a reduction in the quarantine period for fully vaccinated people, a 14 day quarantine period is still required for inbound travellers.

\section{Conclusion}

As the pandemic continues, an appropriate duration of quarantine can help countries with low transmission rates to keep infections at a low level and protect economic and social benefits. A challenge for countries with high risk is to balance the control of local epidemic, the management of imported cases, and the economic recovery. Thus, some counties have shortened the quarantine period to reduce the cost of quarantine and increase the mobility of the population.

Based on scientific evidence and Guangdong's experience, we believe that China's 14 day quarantine period for inbound travellers has been an appropriate cost to control local outbreaks, one of the country's successes in managing the covid-19 pandemic. At the same time, it is necessary to establish effective identification of reinfected and newly infected people to evaluate the risk of local epidemics from inbound travellers with infections that may not have been identified (such as through infection during quarantine or missing a patient). Moreover, the characteristics of SARS-CoV-2 variants (including the incubation period, virus proliferation, herd susceptibility, and vaccine resistance) and the infectivity of vaccinated people need to be monitored to provide data to guide future quarantine strategy.

We thank the Key Research and Development Program of Guangdong Province (2019B111103001) and the National Natural Science Foundation of China (82041030) for financial support.

Contributors and sources: TS developed the original concept, designed the outline of the paper. MZ and HT collected the epidemiological data on covid-19 in China and performed primarily analysis. $\mathrm{HT}, \mathrm{KH}$, and $\mathrm{YZ}$ collected relevant information. $\mathrm{HT}$ and $\mathrm{KH}$ wrote the first draft. $M Z$ and $Y Z$ reviewed the manuscript and contributed to editing. HT and TS completed the writing of the entire manuscript. All authors read and approved the final manuscript.
Competing interests: We have read and understood BMJ policy on declaration of interests and have no relevant interests to declare.

Provenance and peer review: Commissioned; externally peer reviewed.

This article is part of a collection proposed by the Peking University Centre for Public Health and Epidemic Preparedness and Response. Open access fees were funded by individual institutions. The BMJ commissioned, peer reviewed, edited, and made the decision to publish. Li-Ming Li advised on commissioning for this collection. Jin-Ling Tang, Di Wang, and Kamran Abbasi were the lead editors for The BMI.

Hongwei Tu, public health physician ${ }^{1}$

Keqi Hu, research assistant ${ }^{2}$

Meng Zhang, public health physician ${ }^{1}$

Yali Zhuang, public health physician ${ }^{1}$

Tie Song, deputy director ${ }^{1}$

${ }^{1}$ Guangdong Provincial Centre for Diseases Control and Prevention, Guangzhou, China

${ }^{2}$ State Key Laboratory of Organic Geochemistry, Guangzhou Institute of Geochemistry, Chinese Academy of Sciences, Guangzhou, China

Correspondence to:TSong tsong@cdcp.org.cn

\section{(i) (8)} OPEN ACCESS

This is an Open Access article distributed in accordance with the Creative Commons Attribution Non Commercial (CC BY-NC 4.0) license, which permits others to distribute, remix, adapt, build upon this work non-commercially, and license their derivative works on different terms, provided the original work is properly cited and the use is non-commercial. See: http://creativecommons.org/licenses/by-nc/4.0/.

\section{Check for updates}

1 World Health Organization. Considerations for quarantine of contacts of COVID-19 cases: interim guidance, 19 August 2020. WHO, 2020.

2 Russell TW, Wu JT, Clifford S, Edmunds WJ, Kucharski AJ, Jit M, Centre for the Mathematical Modelling of Infectious Diseases COVID-19 working group. Effect of internationally imported cases on internal spread of COVID-19: a mathematical modelling study. Lancet Public Health 2021;6:e12-20. doi:10.1016/S24682667(20)30263-2

3 He X, Lau EHY, Wu P, et al. Temporal dynamics in viral shedding and transmissibility of COVID-19. Nat Med 2020;26:672-5. doi:10.1038/s41591-020-0869-5

4 Meyerowitz EA, Richterman A, Gandhi RT, Sax PE. Transmission of SARS-CoV-2: a review of viral, host, and environmental factors. Ann Intern Med 2021;174:69-79. doi:10.7326/M20-5008 Lauer SA, Grantz KH, Bi Q, et al. The incubation period of coronavirus disease 2019 (covid-19) from publicly reported confirmed cases: estimation and application. Ann Intern Med 2020;172:577-82. doi:10.7326/M20-0504

6 Hu S, Wang W, Wang Y, et al. Infectivity, susceptibility, and risk factors associated with SARS-CoV-2 transmission under intensive contact tracing in Hunan, China. Nat Commun 2021;12:1533. doi:10.1038/s41467-021-21710-6

7 Xin H, Wong JY, Murphy C, et al. The incubation period distribution of coronavirus disease 2019 (COVID-19): a systematic review and meta-analysis. Clin Infect Dis 2021;ciab501. doi:10.1093/cid/ciab501

8 Oran DP, Topol El. The proportion of SARS-CoV-2 infections that are asymptomatic : a systematic review. Ann Intern Med 2021;174:655-62. doi:10.7326/M20-6976 
9 Buitrago-Garcia D, Egli-Gany D, Counotte MJ, et al. Occurrence and transmission potential of asymptomatic and presymptomatic SARSCoV-2 infections: A living systematic review and meta-analysis. PLoS Med 2020;17:e1003346. doi:10.1371/journal.pmed.1003346

10 Zhou B, Thao TTN, Hoffmann D, et al. SARS-CoV-2 spike D614G change enhances replication and transmission. Nature 2021;592:122-7. doi:10.1038/s41586-021-03361-1

11 Arora P, Pöhlmann S, Hoffmann M. Mutation D614G increases SARS-CoV-2 transmission. Signal Transduct Target Ther 2021;6:101. doi:10.1038/s41392-021 00502-w

12 Homma Y, Katsuta T, Oka H, et al. The incubation period of the SARS-CoV-2 B1.1.7 variant is shorter than that of other strains. J Infect 2021;83:e15-7. doi:10.1016/j.jinf.2021.06.011

13 Zhang M, Xiao J, Deng A, et al. Transmission dynamics of an outbreak of the covid-19 delta variant B.1.617.2 Guangdong Province, China, May-June 2021. China CDC Wkly 2021:3:584-6. doi:10.46234/ccdcw2021.148
14 Li B, Deng A, Li K, et al. Viral infection and transmission in a large well-traced outbreak caused by the Delta SARS-CoV-2 variant. medRxiv 2021:2021.07.07.21260122.[Preprint.] doi:10.1101/2021.07.07.21260122

15 Lu J, Peng J, Xiong Q, et al. Clinical, immunological and virological characterization of COVID-19 patients that test re-positive for SARS-CoV-2 by RT-PCR. EBioMedicine 2020;59:102960. doi:10.1016/j. ebiom.2020.102960

16 Ashcroft P, Lehtinen S, Angst DC, Low N, Bonhoeffer S. Quantifying the impact of quarantine duration on COVID-19 transmission. Elife 2021;10:e63704. doi:10.7554/eLife.63704

17 Clifford S, Quilty BJ, Russell TW, et al. Strategies to reduce the risk of SARS-CoV-2 re-introduction from international travellers.medRxiv 2020:2020.07.24.20161281. [Preprint.] doi:10.1101/2020.07.24.20161281.

18 Brooks SK, Webster RK, Smith LE, et al. The psychological impact of quarantine and how to reduce it: rapid review of the evidence. Lancet 2020;395:912 20. doi:10.1016/S0140-6736(20)30460-8
19 Liu Z, VanBlargan LA, Bloyet LM, et al. Identification of SARS-CoV-2 spike mutations that attenuate monoclonal and serum antibody neutralization. Cell Host Microbe 2021:29:477-488 e4 doi:10.1016/j. chom.2021.01.014

20 Hacisuleyman E, Hale C, Saito Y, et al. Vaccine breakthrough infections with SARS-CoV-2 variants. N Engl J Med 2021;384:2212-8. doi:10.1056/ NEJMoa2105000

21 Brown RCH, Kelly D, Wilkinson D, Savulescu J. The scientific and ethical feasibility of immunity passports. Lancet Infect Dis 2021;21:e58-63. doi:10.1016/S1473-3099(20)30766-0

22 Leung K, Wu JT, Leung GM. Effects of adjusting public health, travel, and social measures during the roll-out of COVID-19 vaccination: a modelling study. Lancet Public Health 2021;6:e674-82. doi:10.1016/ S2468-2667(21)00167-5

Cite this as: $B M J$ 2021;375:e066121 http://dx.doi.org/10.1136/bmj-2021-066121 